\title{
In-depth Analysis of Wavelet Transform based Denoising Scheme for Smooth and Textured Images Corrupted with Gaussian Noise
}

\author{
Gopal Prasad \\ ECE Department \\ K. P. Engineering College \\ Agra, UP, India
}

\author{
Arun Kumar Mishra \\ ECE Department \\ K. P. Engineering College \\ Agra, UP, India
}

\author{
Atul Kumar Singh \\ ECE Department \\ Kamla Nehru Institute of Technology \\ Sultanpur, UP, India
}

\begin{abstract}
Image denoising involves the manipulation of the image data to produce a clear and high quality image. Selection of the denoising algorithm is depends on the types of images and applications area of images. Hence, it is necessary to have knowledge about the noise present in the image so as to select the appropriate denoising algorithm. Wavelet based approach is Nobel approach for denoising smooth and textured images corrupted with Gaussian noise. This paper proposed the wavelet based approach with level depending threshold calculated by modified 'sqtwolog' method (universal method) at each scale on the images corrupted with Gaussian noise and performs their in-depth study by considering five major wavelet families like Haar, Daubecheis, Coiflets, Symlets and Biorthogonals. The noisy wavelet coefficients are threshold by Soft Threshold method. The edge preservation and sparse representation abilities of wavelet transform is utilized. A quantitative measure of comparison between original image and denoised image is provided by the PSNR (peak signal to noise ratio) for the smooth and textured images.
\end{abstract}

\section{General Terms}

Image Processing, Gaussian Noise.

\section{Keywords}

Discrete Wavelet Transform (DWT), Wavelet Thresholding, Denoising, Smooth and Textured Images, PSNR (Peak Signal to Noise Ratio).

\section{INTRODUCTION}

Digital images play an important role in the areas of research and technology such as geographical information systems. It is the most vital part in the field of medical science such as ultrasound imaging, X-ray imaging, CT scans, MRI etc. A very large portion of digital image processing includes image compression, denoising and retrieval. Image denoising is a process of removal or reduction of noise that is incurred during the image capturing. Noise comes from blurring as well as due to analog and digital sources. Blurring is the form of bandwidth reduction of images caused by imperfect image formation process such as relative motion between camera and original scene or by an optical system that is out of focus. Noise is in the form of unwanted signal that interferes with the original signal and degrades the visual quality of digital image. The main sources of noise in digital images are imperfect instruments, problem with data acquisition process, interference natural phenomena, transmission and compression. Image denoising forms the preprocessing step in the field of photography, research, technology and medical science, where somehow image has been degraded and needs to be restored before further processing.

Image denoising is a fundamental problem in the field of image processing. Wavelets give a superior performance in image denoising due to properties such as sparsity and multiresolution structure. With Wavelet Transform gaining popularity in the last two decades various algorithms for denoising in wavelet domain were introduced. The focus was shifted from the Spatial and Fourier domain to the Wavelet domain. Ever since Donoho's Wavelet based thresholding approach was published in [1], there was a surge in the denoising papers being published. Although Donoho's concept was not revolutionary, his methods did not require tracking or correlation of the wavelet maxima and minima across the different scales as proposed by Mallat in [2]. Thus, there was a renewed interest in wavelet based denoising techniques since Donoho demonstrated a simple approach to a difficult problem.

Researchers published different ways to compute the parameters for the thresholding of wavelet coefficients. Data adaptive thresholds [3] were introduced to achieve optimum value of threshold. Later efforts found that substantial improvements in perceptual quality could be obtained by translation invariant methods based on thresholding of an undecimated Wavelet Transform [4]. These thresholding techniques were applied to the non-orthogonal wavelet coefficients to reduce artifacts. Multi-wavelets were also used to achieve similar results. Probabilistic models using the statistical properties of the wavelet coefficient seemed to outperform the thresholding techniques and gained ground. Recently, much effort has been devoted to Bayesian denoising in Wavelet domain. Hidden Markov Models and Gaussian Scale Mixtures have also become popular and more research continues to be published.

In this paper in-depth analysis of wavelet transform based denoising scheme for denoising of smooth and textured images which are corrupted by white Gaussian noise is presented. Denoising process is carried out by taking five major wavelets families like Haar, Daubecheis, Coiflets, Symlets and Biorthogonals. The rest of the paper is divided in the various sections. Section 2 briefly explains Gaussian noise. Section 3 presents the Wavelet theory with Wavelet Thresholding. Section 4 presents the proposed denoising approach. Section 5 gives experimental results and analysis. Section 6 gives some conclusions followed by section 7 of references. 


\section{GAUSSIAN NOISE}

Gaussian noise is evenly distributed over the signal. This means that each pixel in the noisy image is the sum of the true pixel value and a random Gaussian distributed noise value. As the name indicates, this type of noise has a Gaussian distribution, which has probability distribution function given by the equation (1) as,

$$
F(g)=\frac{1}{\sqrt{2 \pi \sigma^{2}}} e^{-(g-m)^{2} / 2 \sigma^{2}}
$$

where $g$ represents the gray level, $m$ is the mean or average of the function, and $\sigma$ is the standard deviation of the noise.

\section{WAVELET THEORY}

\subsection{Discrete Wavelet Transform (DWT)}

Wavelets are mathematical functions that analyze data according to scale or resolution [5]. They aid in studying a signal in different windows or at different resolutions. For instance, if the signal is viewed in a large window, gross features can be noticed, but if viewed in a small window, only small features can be noticed.

The term "wavelets" is used to refer to a set of orthonormal basis functions generated by dilation and translation of scaling function $\Phi$ and a mother wavelet $\psi$ [6]. The finite scale multiresolution representation of a discrete function can be called as a discrete wavelet transforms. DWT is a fast linear operation on a data vector, whose length is an integer power of 2. This transform is invertible and orthogonal, where the inverse transform expressed as a matrix is the transpose of the transform matrix. The wavelet basis or function, unlike sines and cosines as in Fourier transform, is quite localized in space. But similar to sines and cosines, individual wavelet functions are localized in frequency.

The orthonormal basis or wavelet basis is defined as

$$
\Psi_{(j, k)}(x)=2^{j / 2} \Psi\left(2^{j} x-k\right)
$$

The scaling function is given as

$$
\Phi_{(j, k)}(x)=2^{j / 2} \Phi\left(2^{j} x-k\right)
$$

where $\Phi$ is called the wavelet function and $j$ and $k$ are integers that scale and dilate the wavelet function. The factor ' $j$ ' in equations (2) and (3) is known as the scale index, which indicates the wavelet's width. The location index $k$ provides the position. The wavelet function is dilated by powers of two and is translated by the integer $k$. In terms of the wavelet coefficients, the wavelet equation is

$$
\Psi(x)=\sum_{k}^{N-1} g_{k} \sqrt{2 \Phi(2 x-k)}
$$

where $g_{0}, g_{l}, g_{2} \ldots$ in equation (4) are high pass wavelet coefficients. Writing the scaling equation in terms of the scaling coefficients as given below, we get,

$$
\Phi(x)=\sum_{k}^{N-1} h_{k} \sqrt{2 \Phi(2 x-k)}
$$

In equation (5) the function $\Phi(\mathrm{x})$ is the scaling function and the coefficients $h_{0}, h_{1}, h_{2} \ldots$ are low pass scaling coefficients. The wavelet and scaling coefficients are related by the quadrature mirror relationship, which is

$$
g_{n}=(-1)^{n} h_{1-n+N}
$$

The term $N$ is the number of vanishing moments in equation (6). A graphical representation of DWT is shown in Fig. 1. Note that, $Y_{0}$ is the initial signal.

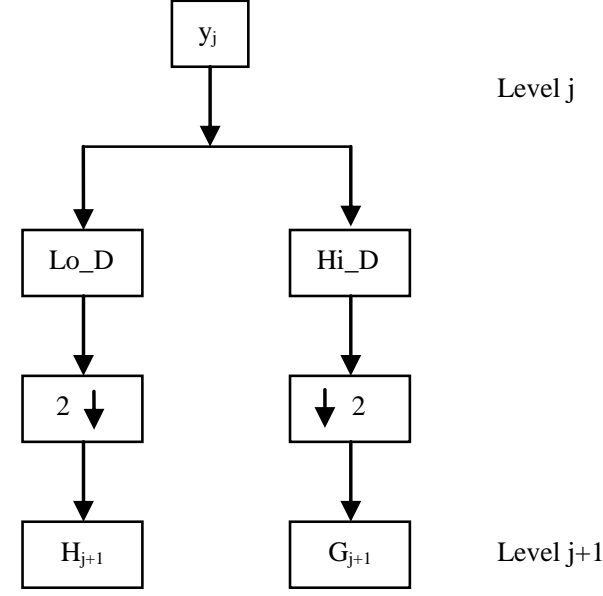

Fig. 1. A 1-Dimensional DWT - Decomposition step

Wavelets are classified into a family by the number of vanishing moments $N$. Within each family of wavelets there are wavelet subclasses distinguished by the number of coefficients and by the level of iterations [7]. The filter lengths and the number of vanishing moments for five different wavelet families are tabulated in Table 1.

Table 1 Wavelet families and their properties

\begin{tabular}{|c|c|c|}
\hline $\begin{array}{c}\text { Wavelet } \\
\text { Family }\end{array}$ & Filters length & $\begin{array}{c}\text { Number of vanishing } \\
\text { moments, } \boldsymbol{N}\end{array}$ \\
\hline Haar & 2 & 1 \\
\hline Daubechies & $2 \mathrm{~N}$ & $\mathrm{~N}$ \\
\hline Coiflets & $6 \mathrm{~N}$ & $\mathrm{~N}-1$ \\
\hline Symlets & $2 \mathrm{~N}$ & $\mathrm{~N}$ \\
\hline Biorthogonal & $\begin{array}{c}\max (2 \mathrm{Nr}, 2 \mathrm{Nd})+2 \text { but } \\
\text { essentially }\end{array}$ \\
\hline
\end{tabular}

\subsection{Wavelet Thresholding}

The term wavelet thresholding performs the decomposition of the data or the image into wavelet coefficients. It compares the detail coefficients with a given threshold value, and shrinking these coefficients close to zero to take away the effect of noise in the data. The image is reconstructed from the modified coefficients. This process is also known as the inverse discrete wavelet transform. During thresholding, a wavelet coefficient is compared with a given threshold and is set to zero if its magnitude is less than the threshold; otherwise, it is retained or modified depending on the threshold rule.

The hard-thresholding $T_{H}$ can be defined by equation (7) as

$$
T_{H}=\left\{\begin{array}{l}
x \text { for }|x| \geq t \\
0 \text { for all other regions }
\end{array}\right.
$$

Here $t$ is the threshold value. A plot of $T_{\mathrm{H}}$ is shown in Fig. 2.

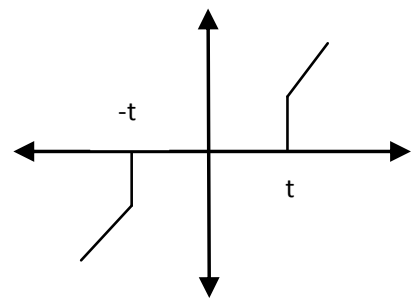

Fig. 2. Hard thresholding 
Thus, all coefficients whose magnitude is greater than the selected threshold value $(t)$ remain as they are and the others with magnitudes smaller than tare set to zero. It creates a region around zero where the coefficients are considered negligible.

Soft thresholding is where the coefficients with greater than the threshold are shrunk towards zero after comparing them to a threshold value. It is defined by equation (8) as

$$
T_{S}= \begin{cases}\operatorname{sign}(x)(|x|-t) & \text { for }|x|>t \\ 0 & \text { for all other regions }\end{cases}
$$

Here $t$ is the threshold value. A plot of $T_{\mathrm{S}}$ is shown in Fig. 3 .

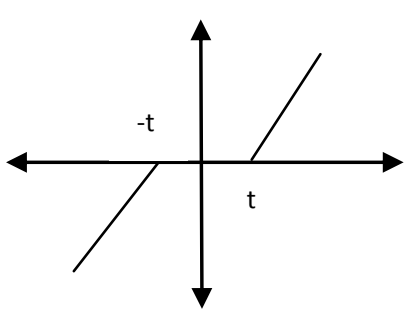

Fig. 3.Soft thresholding

In practice, it can be seen that the soft threshold method is much better and yields more clear and high quality smooth and textured images. This is because the hard method is discontinuous and yields abrupt artifacts in the recovered images. Also, the soft thresholding method yields a smaller minimum mean squared error compared to hard form of thresholding.

\section{PROPOSED APPROACH}

Let the original image (2D signal) be represented by, $I(i, j)$. The noisy $\operatorname{Image}_{n}(i, j)$ is given by, $I_{n}(i, j)=I(i, j)+$ $\sigma . Z_{g}(i, j)$, Where, $\sigma$ is the noise standard deviation and $Z_{g}(i, j)$ is the white noise of zero mean $\mu_{g}=0$ and unit variance $\left(\sigma_{g}^{2}=1\right)$. Here the objective is to obtain the best estimate $I_{d}(i, j)$ of noisy image $I_{n}(i, j)$ and binarize the denoised image $I_{d}(i, j)$ to achieve its binary version $I_{d b i n}(i, j)$.

The wavelet based scheme for denoising is shown in Fig.4.The scheme has following main steps,

a) Find level-4 wavelet transform of noisy image $I_{n}(i, j)$.

b) Calculate noise standard deviation $(\sigma)$ and estimate threshold $(\lambda)$ for each level.

c) Perform Soft thresholding of wavelet coefficients at each level of decomposition.

d) Perform level-4 inverse wavelet transform of thresholded wavelet coefficients to get denoised image $I_{d}(i, j)$.

e) Obtain binary image $I_{d b i n}(i, j)$ of denoised image $I_{d}(i, j)$ by global Otsu method.

f) Find the evaluation parameters between binary version of original noise free image $I_{d b i n}(i, j)$ and to observe the quality of denoising.
The threshold $(\lambda)$ used is the Universal Threshold [8] for thresholding, which is given by equation (9) as,

$$
\lambda_{j}=\sigma \sqrt{2 \log \left(N_{j}\right)}
$$

Where, $\left(N_{j}\right)$ is the size of the wavelet coefficient matrix at $j^{t h}$ level and $\sigma$ is the noise standard deviation. The value of noise standard deviation can be calculated by Median Absolute Deviation (MAD) of high frequency wavelet coefficients $\left(\mathrm{C}_{\mathrm{H}}, \mathrm{C}_{\mathrm{V}}\right.$ and $\mathrm{C}_{\mathrm{D}}$, in case of an image) of noisy image at level 1 of decomposition. It is given by equation (10) as,

$$
\sigma=\frac{M A D}{0.6745}=\frac{\operatorname{median}\left(\left|\omega_{k}\right|\right)}{0.6745}
$$

Where, $\omega_{k}$ represent wavelet coefficients at scale 1 [8]. In the proposed scheme, the input noisy images are decomposed in 4 levels and the threshold for each level is found then wavelet coefficients are soft thresholded to get the denoised smooth and textured image.

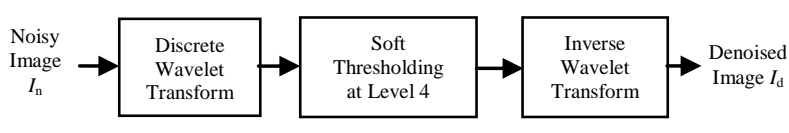

Fig.4. Wavelet based approach

\section{RESULTS AND ANALYSIS}

\subsection{Peak Signal to Noise Ratio (PSNR)}

The experimental evaluation is performed on two smooth images like "Lena" and "Pepper" of size 512 X 512 pixels and two textured images like "Textured1" and "Textured2"of size 512 X 512 pixels at different noise levels using wavelet families like Haar, Daubecheis, Coiflets, Symlets and Biorthogonals up to level 4 decomposition. The objective quality [9] of the reconstructed image is measured by equation (11) as,

$$
P S N R=10 \log _{10} \frac{255^{2}}{m s e}
$$

where $m s e$ is the mean square error between the original (i.e. $x$ ) and the de-noised image (i.e. ) with size $M \times N$ can be expressed by equation (12) as,

$$
m s e=\frac{1}{M \times N} \sum_{i=1}^{M} \sum_{j=1}^{N}[x(i, j)-\hat{x}(i, j)]^{2}
$$

Below table 2 and table 3 shows PSNR of "Lena" and "Pepper" images at different noise levels using different wavelet families. Table 4 and table 5 shows PSNR of "Textured1" and "Textured2" images at different noise levels using different wavelet families. 
Table 2 PSNR (dB) of "Lena" image up to level 4 decomposition (L1, L2, L3, L4).

\begin{tabular}{|c|c|c|c|c|c|c|c|c|c|c|c|c|c|c|c|c|}
\hline \multirow{2}{*}{$\begin{array}{l}\text { Types of } \\
\text { Wavelets }\end{array}$} & \multicolumn{4}{|c|}{$\sigma=10$} & \multicolumn{4}{|c|}{$\sigma=20$} & \multicolumn{4}{|c|}{$\sigma=\mathbf{3 0}$} & \multicolumn{4}{|c|}{$\sigma=40$} \\
\hline & L1 & L2 & L3 & L4 & L1 & L2 & $\mathbf{L 3}$ & L4 & L1 & L2 & L3 & L4 & L1 & L2 & L3 & L4 \\
\hline Haar & 29.91 & 30.43 & 30.47 & 30.49 & 23.24 & 23.55 & 23.59 & 23.63 & 19.50 & 19.69 & 19.77 & 19.76 & 16.98 & 17.10 & 17.16 & 17.17 \\
\hline db10 & 30.14 & 30.67 & 30.73 & 30.70 & 23.29 & 23.60 & 23.67 & 23.67 & 19.54 & 19.76 & 19.80 & 19.76 & 16.99 & 17.17 & 17.19 & 17.19 \\
\hline Coif5 & 30.18 & 30.69 & 30.76 & 30.79 & 23.33 & 23.63 & 23.68 & 23.69 & 19.55 & 19.74 & 19.79 & 19.78 & 17.01 & 17.15 & 17.18 & 17.17 \\
\hline Bior6.8 & 30.20 & 30.68 & 30.82 & 30.81 & 23.34 & 23.62 & 23.70 & 23.69 & 19.57 & 19.75 & 19.76 & 19.78 & 17.03 & 17.17 & 17.17 & 17.20 \\
\hline Sym4 & 30.14 & 30.66 & 30.74 & 30.74 & 23.30 & 23.59 & 23.66 & 23.65 & 19.54 & 19.74 & 19.79 & 19.78 & 17.01 & 17.17 & 17.18 & 17.20 \\
\hline
\end{tabular}

For $\sigma=20$, Bior6.8, level 4 decomposition (Lena)

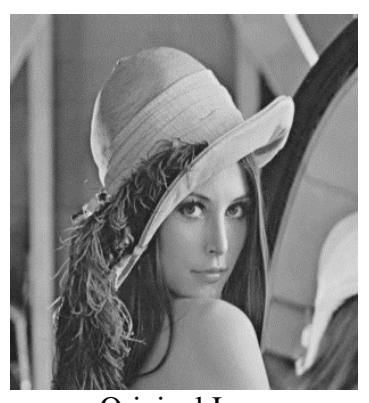

Original Image

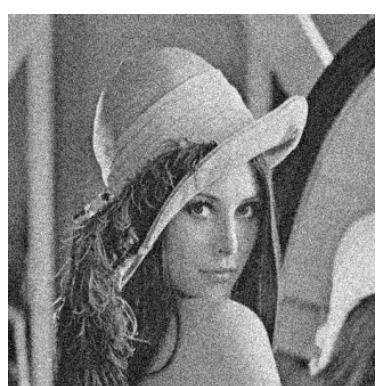

Noisy Image

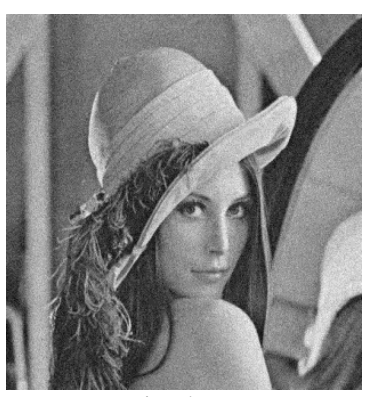

Denoised Image

Table 3 PSNR (dB) of "Pepper" image up to level 4 decomposition (L1, L2, L3, L4).

\begin{tabular}{|c|c|c|c|c|c|c|c|c|c|c|c|c|c|c|c|c|}
\hline \multirow{2}{*}{$\begin{array}{l}\text { Types of } \\
\text { Wavelet }\end{array}$} & \multicolumn{4}{|c|}{$\square=\square \square$} & \multicolumn{4}{|c|}{$\square=\square \square$} & \multicolumn{4}{|c|}{$\square=\square \square$} & \multicolumn{4}{|c|}{$\square=\square \square$} \\
\hline & L1 & L2 & L3 & L4 & L1 & L2 & L3 & L4 & L1 & L2 & L3 & L4 & L1 & L2 & L3 & L4 \\
\hline Haar & 27.37 & 27.56 & 27.60 & 27.66 & 21.40 & 21.58 & 21.60 & 21.61 & 18.37 & 18.51 & 18.54 & 18.55 & 16.27 & 16.38 & 16.41 & 16.38 \\
\hline db10 & 27.37 & 27.58 & 27.64 & 27.62 & 21.41 & 21.57 & 21.60 & 21.61 & 18.35 & 18.53 & 18.55 & 18.55 & 16.27 & 16.39 & 16.40 & 16.40 \\
\hline Coif5 & 27.32 & 27.60 & 27.63 & 27.62 & 21.38 & 21.60 & 21.62 & 21.63 & 18.38 & 18.51 & 18.55 & 18.55 & 16.26 & 16.39 & 16.41 & 16.39 \\
\hline $\begin{array}{l}\text { Bior6.8 } \\
\end{array}$ & 27.37 & 27.56 & 27.65 & 27.66 & 21.40 & 21.56 & 21.62 & 21.59 & 18.37 & 18.52 & 18.54 & 18.54 & 16.27 & 16.37 & 16.41 & 16.41 \\
\hline Sym4 & 27.40 & 27.62 & 27.62 & 27.63 & 21.39 & 21.58 & 21.63 & 21.63 & 18.38 & 18.51 & 18.53 & 18.54 & 16.24 & 16.39 & 16.41 & 16.41 \\
\hline
\end{tabular}

For $\sigma=30$, Coif5, level 4 decomposition (Pepper)

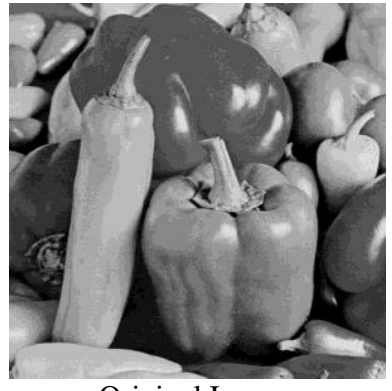

Original Image

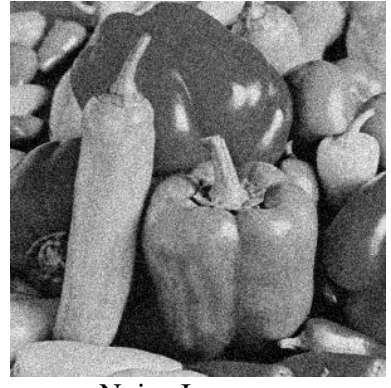

Noisy Image

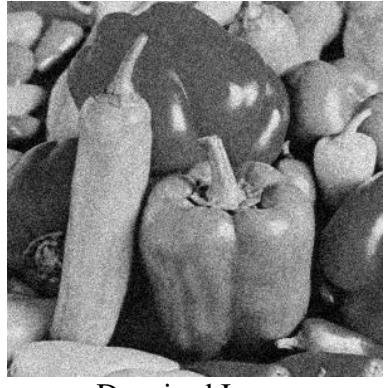

Denoised Image

Table 4 PSNR (dB) of “Textured1" image up to level 4 decomposition (L1, L2, L3, L4).

\begin{tabular}{|c|c|c|c|c|c|c|c|c|c|c|c|c|c|c|c|c|}
\hline \multirow{2}{*}{$\begin{array}{l}\text { Types of } \\
\text { Wavelet } \\
\end{array}$} & \multicolumn{4}{|c|}{$\square=\square \square$} & \multicolumn{4}{|c|}{$\square=\square \square$} & \multicolumn{4}{|c|}{$\square=\square \square$} & \multicolumn{4}{|c|}{$\square=\square \square$} \\
\hline & L1 & L2 & L3 & L4 & L1 & L2 & L3 & L4 & L1 & L2 & L3 & L4 & L1 & L2 & L3 & L4 \\
\hline Haar & 24.70 & 24.59 & 24.55 & 24.59 & 20.99 & 20.98 & 20.95 & 20.95 & 18.47 & 18.47 & 18.48 & 18.47 & 16.55 & 16.57 & 16.54 & 16.54 \\
\hline db10 & 24.75 & 24.67 & 24.64 & 24.62 & 21.03 & 21.02 & 21.01 & 21.02 & 18.49 & 18.55 & 18.51 & 18.52 & 16.58 & 16.60 & 16.58 & 16.60 \\
\hline Coif5 & 24.73 & 24.70 & 24.67 & 24.63 & 21.06 & 21.04 & 21.04 & 21.02 & 18.53 & 18.55 & 18.51 & 18.51 & 16.58 & 16.60 & 16.60 & 16.58 \\
\hline Bior6.8 & 24.75 & 24.65 & 24.64 & 24.64 & 21.05 & 21.04 & 21.03 & 21.02 & 18.51 & 18.53 & 18.52 & 18.52 & 16.58 & 16.60 & 16.60 & 16.60 \\
\hline Sym4 & 24.73 & 24.70 & 24.63 & 24.61 & 21.04 & 21.02 & 21.00 & 21.02 & 18.51 & 18.52 & 18.50 & 18.51 & 16.57 & 16.57 & 16.58 & 16.60 \\
\hline
\end{tabular}


For $\sigma=20$, Sym4, level 2 decomposition (Textured1)

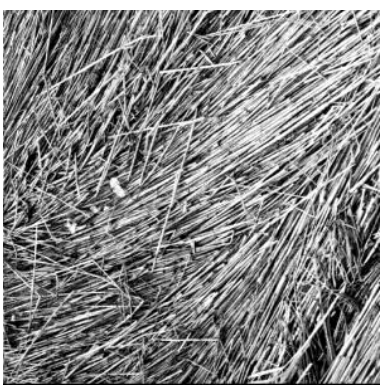

Original Image

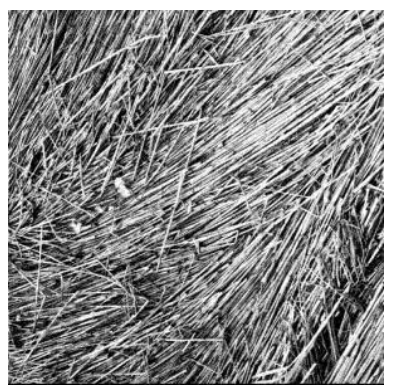

Noisy Image

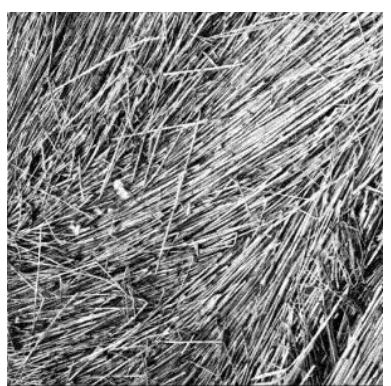

Denoised Image

Table 5 PSNR (dB) of "Textured2" image up to level 4 decomposition (L1, L2, L3, L4).

\begin{tabular}{|c|c|c|c|c|c|c|c|c|c|c|c|c|c|c|c|c|}
\hline \multirow{2}{*}{$\begin{array}{l}\text { Types of } \\
\text { Wavelet }\end{array}$} & \multicolumn{4}{|c|}{$\square=\square \square$} & \multicolumn{4}{|c|}{$\square=\square \square$} & \multicolumn{4}{|c|}{$\square=\square \square$} & \multicolumn{4}{|c|}{$\square=\square \square$} \\
\hline & L1 & L2 & $\mathbf{L 3}$ & L4 & L1 & L2 & L3 & L4 & L1 & L2 & L3 & L4 & L1 & L2 & L3 & L4 \\
\hline Haar & 24.88 & 24.79 & 24.77 & 24.77 & 21.07 & 21.08 & 21.05 & 21.06 & 18.51 & 18.55 & 18.52 & 18.51 & 16.57 & 16.58 & 16.59 & 16.57 \\
\hline db10 & 24.88 & 24.83 & 24.77 & 24.80 & 21.10 & 21.06 & 21.08 & 21.09 & 18.53 & 18.54 & 18.55 & 18.55 & 16.56 & 16.63 & 16.56 & 16.63 \\
\hline Coif5 & 24.91 & 24.82 & 24.82 & 24.76 & 21.11 & 21.12 & 21.09 & 21.08 & 18.52 & 18.54 & 18.57 & 18.56 & 16.57 & 16.64 & 16.61 & 16.60 \\
\hline Bior6.8 & 24.89 & 24.78 & 24.79 & 24.81 & 21.11 & 21.09 & 21.10 & 21.12 & 18.53 & 18.54 & 18.55 & 18.56 & 16.61 & 16.61 & 16.59 & 16.59 \\
\hline Sym4 & 24.88 & 24.84 & 24.79 & 24.80 & 21.10 & 21.10 & 21.10 & 21.08 & 18.50 & 18.56 & 18.56 & 18.53 & 16.57 & 16.61 & 16.62 & 16.60 \\
\hline
\end{tabular}

For $\boldsymbol{\sigma}=30$, Coif5, level 2 decomposition (Textured2)

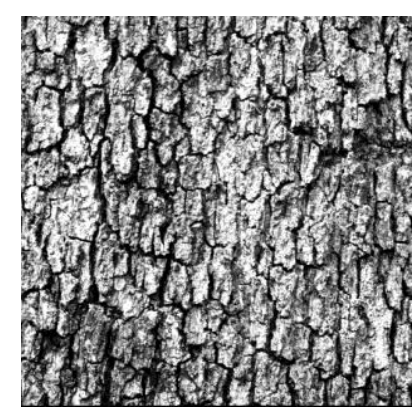

Original Image

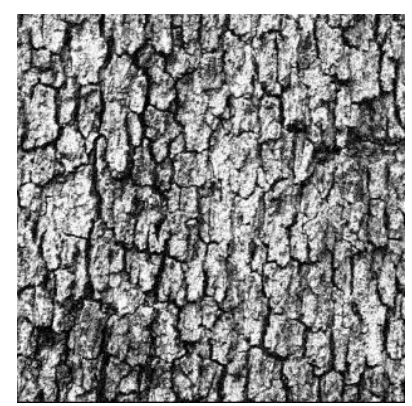

Noisy Image

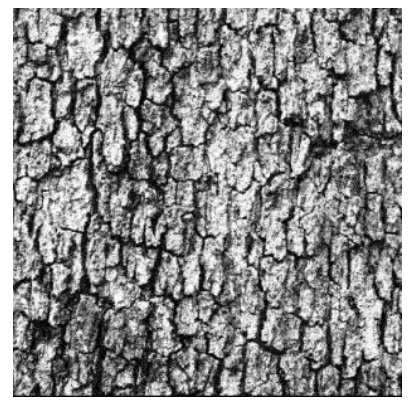

Denoised Image

\section{CONCLUSION}

A large number of wavelet based image denoising methods along with several types of thresholding have been proposed in recent years. These methods are mainly reported for images such as Lena, Barbara etc. The general methods based on wavelet transform using soft thresholding are best capable of preserving edges and fine details and therefore, suitable for denoising of smooth and textured images.

From the experimental and mathematical results it can be concluded that PSNR is basically a comparison between original and de-noised image as how the de-noised image is close to original image. Tables 2 and 3 shows the peak signal to noise ratio (PSNR) of smooth images like 'Lena' and 'Pepper' by using wavelet families like Haar, Daubecheis, Coiflets, Symlets and Biorthogonals at level 4 decomposition. Similarly tables 4 and 5 shows the peak signal to noise ratio (PSNR) of textured images like 'Textured1' and 'Textured2' by using wavelet families like Haar, Daubecheis, Coiflets, Symlets and Biorthogonals at level 4 decomposition. From the tables 2, 3, 4, 5 we can conclude that

1. Coif5 and bior6.8 wavelets results high PSNR against haar, db10 andsym4 wavelets for Smooth images like Lena and Pepper.
2. For Textured images Coif5 and sym4 gives best results.

3. Smooth images results high PSNR against Textured images.

4. Decomposition up to level 4 is the saturation level for Smooth images and decomposition up to level 2 is the saturation level for textured images.

5. For Textured images if $\sigma$ is low $(\sigma=10,15,20)$, level 1 decomposition results high PSNR but for large value of $\sigma$ $(\sigma=30,35,40)$, level 2 decomposition gives high PSNR.

\section{REFERENCES}

[1] D. L. Donoho, "De-noising by soft-thresholding", IEEE Trans. Information Theory, vol.41, no.3, pp.613- 627, May1995.

http://wwwstat.stanford.edu/ donoho/Reports/1992/deno isereleas e3.ps.Z.

[2] S. G. Mallat and W. L. Hwang, "Singularity detection and processing with wavelets," IEEE Trans. Inform. Theory, vol. 38, pp. 617-643, Mar. 1992.

[3] David L. Donoho and Iain M. Johnstone, "Adapting to Unknown Smoothness via Wavelet Shrinkage," Journal 
of American StatisticalAssociation, 90(432):1200-1224, December 1995.

[4] R. Coifman and D. Donoho, "Translation invariant denoising," in Lecture Notes in Statistics: Wavelets and Statistics, vol. New York: Springer-Verlag, pp. 125-150, 1995.

[5] Amara Graps, "An Introduction to Wavelets," IEEE Computational Science and Engineering, summer 1995, Vol 2, No. 2.
[6] Matlab 7.8, "Wavelet tool box,".

[7] Matlab7.8, "Matlab," http://www.mathworks.com/, May 2009.

[8] D. L. Donoho, "De-noising by soft thresholding", IEEE Transaction on Information Theory, Vol. 41, pp. 613627, 1995.

[9] Image Processing Fundamentals-Statistics, "Signal to Noise ratio," http://www.ph.tn.tudelft.nl/courses/FIP/no frames/fip-Statisti .html, 2001. 